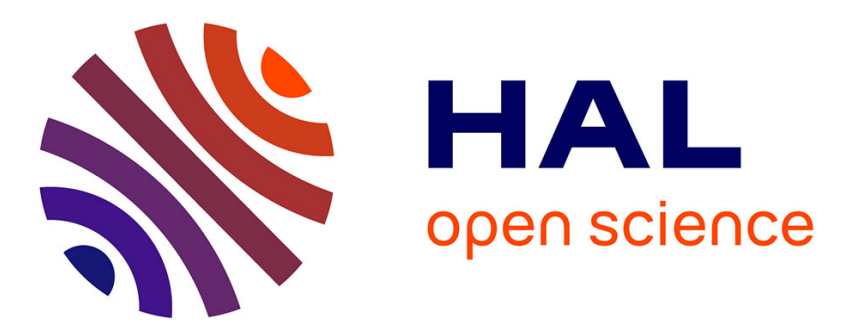

\title{
Les infrastructures de transport et la logique financière du partenariat public-privé: quelques paradoxes
}

\author{
Alain Bonnafous
}

\section{To cite this version:}

Alain Bonnafous. Les infrastructures de transport et la logique financière du partenariat publicprivé: quelques paradoxes. Revue Française d'Economie, 2002, XVII (1), pp. 173-194. 10.3406/rfeco.2002.1458 . halshs-00079845

\section{HAL Id: halshs-00079845 \\ https://shs.hal.science/halshs-00079845}

Submitted on 11 Apr 2007

HAL is a multi-disciplinary open access archive for the deposit and dissemination of scientific research documents, whether they are published or not. The documents may come from teaching and research institutions in France or abroad, or from public or private research centers.
L'archive ouverte pluridisciplinaire $\mathbf{H A L}$, est destinée au dépôt et à la diffusion de documents scientifiques de niveau recherche, publiés ou non, émanant des établissements d'enseignement et de recherche français ou étrangers, des laboratoires publics ou privés. 


\title{
Les infrastructures de transport et la logique financière du partenariat public - privé : quelques paradoxes
}

\author{
Alain Bonnafous \\ Professeur \\ Université Lumière-Lyon 2, IEP de Lyon
}

Laboratoire d'Economie des Transport

69363 Lyon Cedex 07 - France

alain.bonnafous@let.ish-lyon.cnrs.fr

(Article publié dans le $\mathrm{n}^{\circ}$ de juillet 2002 de la Revue Française d'Economie)

Cet article est inspiré par quelques questions vives que pose la pratique du partenariat public-privé dans le champ des nouvelles infrastructures de transport, en particulier la logique financière de ce partenariat. Il peut toutefois concerner, comme cela arrive lorsqu'une problématique sectorielle est soulevée, tous les domaines d'activité qui ont à connaître des problèmes liés au financement, à la construction et à l'exploitation d'infrastructures publiques, dès lors que l'administration peut trouver avantage à impliquer un partenaire privé dans ces trois volets en dépit du fait que les immobilisations ont un caractère évident de bien public.

Cela nous renvoie à la très ancienne pratique de la concession, contrat par lequel le concédant charge un opérateur généralement privé d'exécuter un ouvrage et d'en assurer l'exploitation au moyen de redevances perçues sur les usagers. Selon la terminologie internationale devenue courante, on parle plutôt aujourd'hui d'une pratique de BOT : dans ce système "Build, Operate and Transfer », l'opérateur finance en tout ou partie et construit l'infrastructure qu'il exploite pendant une durée limitée (15 à 75 ans dans le domaine des transports). A l'issue de cette période, l'infrastructure devient propriété des pouvoirs publics. C'est dans ce cas que se situe l'analyse qui va suivre.

\section{Un renouveau mondial, mais parfois paradoxal, des pratiques de concession}

Contrairement à ce qu'ont pu suggérer certaines mésaventures financières comme celles du tunnel sous la Manche ou d'Orlyval ${ }^{1}$, l'implication d'opérateurs privés dans la réalisation ou l'exploitation d'infrastructures de transport n'est pas en voie de disparition. Pour rester dans le domaine ferroviaire, le regard serait trompeur s'il était limité aux réseaux européens qui comptent, avec Railtrack au Royaume Uni, une seule société privée propriétaire d'un grand réseau d'infrastructure. Dans une publication récente, K.J. Budin et I.S.Thomson (2001) font état de concessions ou de réalisations ferroviaires confiées à un opérateur privé qui concernent plusieurs dizaines de pays sur tous les continents, alors que de telles configurations étaient anecdotiques dans les années 80 .

\footnotetext{
${ }^{1}$ Dans le cas de l'Eurotunnel, la faillite de l'opérateur privé, qui devait résulter d'une dérive considérable des coûts, n'a pu être évitée que par des ajustements financiers complexes et un allongement de la durée de la concession. Quant au système Orlyval qui relie l'aéroport d'Orly à la ligne B du RER, l'insuffisance de ses recettes commerciales a conduit le consortium privé qui en était l'opérateur à le remettre, pour le franc symbolique, à la RATP.
} 
En dépit d'une tradition de quelques 70 ans d'ancrage du rail dans la sphère publique, la France elle-même ne sera pas épargnée, à ses frontières, par cette évolution : il est dores et déjà acquis que, sous la pression des dispositions communautaires, la liaison ferroviaire à grande vitesse entre Perpignan et Figueras fera l'objet d'un appel d'offre ouvert aux opérateurs privés et il en sera certainement de même du tunnel de base sous le Mont Cenis, élément central et fort coûteux de la liaison ferroviaire entre Lyon et Turin.

Relevons ce que ce scénario peut avoir d'étrange au premier abord: alors que la très bonne rentabilité financière de grands projets comme les TGV Sud-Est et Atlantique permettait d'en assurer l'autofinancement, il ne venait à l'idée de personne qu'ils puissent être réalisés et exploités autrement que par l'opérateur public ; bien que les deux franchissements précités ont une rentabilité financière modeste et requièrent donc une part importante de financement public, l'implication d'opérateurs privés dans leur financement et leur réalisation n'est guère contestée.

Les exemples d'intervention d'investisseurs privés, et plus encore les projets, abondent également en matière de ponts ${ }^{2}$, d'installations aéroportuaires ou de ports (Baird, 2001). Le secteur routier n'est pas en reste dans cette évolution.

En France, les expériences récentes d'ouvrages autoroutiers en milieu urbain, avec le demi échec de TEO à Lyon et la relative réussite du tunnel Prado - Carénage à Marseille, révèlent, certes, des difficultés dans la mise en œuvre de tels partenariats mais ne les disqualifient pas pour autant.

En ce domaine, le dispositif français de financement autoroutier a plusieurs décennies d'expérience à faire valoir. Il vient d'être réformé pour cause d'incompatibilité avec la législation européenne et cette réforme va dans le sens d'une intervention plus importante de partenaires privés. Ce dispositif reposait, en effet, sur un système dit d'adossement, qui consistait à confier au concessionnaire d'un large réseau d'autoroutes la construction et l'exploitation d'une section complémentaire de ce réseau. Le financement de cette nouvelle section, en principe moins rentable que les précédentes ${ }^{3}$, était d'abord assuré par les cashflows des anciennes sections puis par un emprunt levé par la société concessionnaire mais garanti par l'Etat et souvent gagé sur un allongement des concessions existantes.

Le nouveau système mis en place résulte, en somme, de la transposition de la directive communautaire 93-37 et abolit la discrimination dont bénéficiaient jusqu'ici les sociétés d'autoroutes installées mais, du même coup, il abolit également ce mode de financement, fort commode pour la puissance publique et par le biais duquel un nouveau tronçon pouvait être financé par les profits que dégageaient les éléments de réseau déjà amortis. C'est ainsi que les nouveaux appels à concession voient apparaître des opérateurs privés qui, jusqu'ici, étaient hors jeu, mais aussi des subventions d'équilibre qui, auparavant, n'étaient pas nécessaires ${ }^{4}$ car elles pouvaient être camouflées dans les recettes à venir de tronçons financièrement amortis.

Ainsi voit-on se préciser, là encore, une chronologie paradoxale. Entre 1956 et 1963, naissent cinq sociétés d'économie mixte concessionnaires d'autoroutes, d'où leur dénomination de SEMCA. Elles ne comportaient, en réalité, que des capitaux publics, mais leur relatif succès a conduit l'Etat à attribuer des concessions au secteur privé. Quatre sociétés ont ainsi été créées entre 1970 et 1973, dont les actionnaires étaient des banques et des

\footnotetext{
${ }^{2}$ L'imposant viaduc de Millau fait l'objet d'une concession à un consortium privé qui a été « bouclée » en 2001.

${ }^{3}$ Pour autant que les éléments du réseau aient été construit dans un ordre de rentabilité décroissante, ce à quoi la Direction des Routes et le ministère des finances ont généralement veillé.

${ }^{4}$ Le premier cas traité dans le nouveau dispositif (et ne résultant pas d'une annulation d'une concession accordée sous l'ancien) est celui de la section Rouen - Alençon de l'autoroute A28 long de $120 \mathrm{~km}$. Il a été concédé à un groupement privé (Bouygues, EGIS et CDC) et prévoit une subvention d'équilibre d'environ $50 \%$ du coût de l'investissement.
} 
entreprises de travaux publics. Le succès de ce début de privatisation sera compromis par les effets du choc pétrolier de 1973 et seule COFIROUTE survivra comme société privée ${ }^{5}$.

C'est ainsi un secteur dominé par les opérateurs publics qui s'ouvre aujourd'hui à la concurrence d'opérateurs privés. C'est là une nouvelle version d'un paradoxe, dont nous trouverons plus loin un écho théorique, qui veut que les premiers tronçons, c'est à dire les plus rentables, aient été construits et exploités par des entreprises publiques et que ceux qui restent à réaliser devraient être significativement ouverts à des entreprises privées. Mais cette inflexion s'inscrit dans une tendance au développement des partenariats public-privé qui déborde de beaucoup le seul cas français.

\section{Quelques explications}

Cette tendance au recours à des opérateurs privés a ses raisons. Elles ont été exprimées dans de nombreuses publications qui évoquent les diverses justifications de l'implication croissante des opérateurs privés dans un domaine principalement dévolu à la sphère publique jusqu'à une période récente.

La première famille de raisons, le plus souvent évoquée, tient à la possibilité, pour un opérateur privé, de mieux gérer la construction et le fonctionnement du projet considéré. Cela revient à supposer que le taux de rentabilité interne (TRI) propre au projet n'est pas le même selon que la gestion est assurée par une administration ou un établissement public ou par une entreprise théoriquement familiarisée avec les efforts de bonne gestion. De multiples explications de cette différence sont avancées : des salaires moins élevés dans le secteur privé pour certaines catégories de personnels, une plus grande flexibilité avec des dirigeants et autres salariés mieux responsabilisés, des délais de construction plus rapides qui hâtent le retour sur investissement ou, encore, une plus grande capacité à résister à des demandes politiques impliquant des coûts supplémentaires.

Cette supériorité des entreprises privées dans l'efficacité de la gestion relève d'une très ancienne controverse alimentée par de flagrantes crispations idéologiques. Cependant, à mesure que se sont accumulées des statistiques qui combinent les séries croisées et les séries chronologiques issues de comptabilités quasiment standardisées, les analyses se sont accumulées qui concluent à une différence significative en faveur du secteur privé. L'une des plus récentes et des plus complètes de ces analyses, proposée par K.L. Dewenter et P.H. Malatesta (2001), concerne 1369 entreprises de plus de 500 salariés à travers le monde pour des observations qui vont de 1975 à 1995. Elle ne laisse guère de doute sur la significativité des résultats.

La deuxième série de justification est particulièrement importante dans les pays peu accoutumés aux infrastructures à péage. J.A. Gomez-Ibanez et J.R. Meyer (1993) font observer qu'un péage est mal accepté lorsque l'Etat est propriétaire de l'infrastructure alors que cela paraît naturel lorsque l'ouvrage est financé par une entreprise privée. Cette solution est ainsi souvent la seule qui permette d'appliquer le principe " usager - payeur ». Il est à noter qu'une telle culture, qui admet cette évidence qu'un entrepreneur privé peut voir son investissement rétribué, est typiquement anglo-saxone.

La troisième série de justifications du recours à un financement privé tient à l'excès d'endettement public. Cet excès d'endettement peut concerner l'opérateur public susceptible d'assumer le projet ou le pays lui-même. Dans le premier cas, même si la dette liée à un projet est gagée sur les recettes à venir, l'endettement supplémentaire peut avoir des inconvénients en matière de cotation financière de cet opérateur public. Plus généralement, le gouvernement concerné peut avoir un objectif de maîtrise de la dette publique. Par exemple, les pays européens de l'Union Economique et Monétaire ont du respecter un critère de convergence

\footnotetext{
${ }^{5}$ AREA sera transformée en SEMCA ; APPEL et ACOBA seront absorbées par deux autres SEMCA.
} 
relatif à la dette publique (au plus $60 \%$ du PNB). Indépendamment de ce cas particulier ${ }^{6}$, tout pays dont la dette publique est élevée peut souhaiter se libérer de " l'effet boule de neige » par lequel la charge de la dette vient abonder le poids de la dette dès lors que les taux d'intérêt sont supérieurs au taux de croissance nominale.

Ces séries de raisons peuvent évidemment se conjuguer mais peuvent aussi ne pas suffire à compenser le surcoût éventuel pour les finances publiques de la solution privée, ainsi que le reconnaît G. Mills (1996). Il rejoint beaucoup d'auteurs qui récusent un choix systématique pour l'une ou l'autre des solutions et suggèrent plutôt de déterminer au cas par cas la solution la mieux appropriée aux circonstances.

Cet article ne concerne pas les deux premières familles de raisons (la bonne gestion et l'acceptabilité du péage), mais seulement la troisième : quelles sont les conditions réelles d'une réduction du financement public ? Quelles sont les conséquences sur le niveau de ce financement public d'une faible rentabilité financière selon que l'opérateur est public ou privé ? Le recours aux opérateurs privés n'est-il pertinent que lorsque la rentabilité financière est très élevée?

Sur la base de quelques réponses à ces questions, nous précisons leurs conséquences en matière de choix politiques, en particulier les conséquences de ce que nous appelons le paradoxe du taux de rentabilité, dont nous verrons qu'il est cohérent avec le retour en grâce du partenariat public - privé.

\section{Le besoin de subvention}

Dans l'analyse qui suit, les prix et les coûts, ainsi que le surplus collectif et celui des usagers, sont par convention supposés indépendants du choix de l'opérateur public ou privé. Nous verrons à la fin de cet article ce que peut impliquer la levée de cette hypothèse.

Pour simplifier les choses dans un premier temps, en particulier pour faciliter la formalisation du problème, nous nous en tiendrons ainsi à une situation "stylisée" caractérisée par les hypothèses suivantes :

- Dans l'option dite "publique", l'opérateur en charge du projet est supposé ne pas réaliser de profits mais il est réputé couvrir les coûts d'investissement et d'exploitation, y compris les charges financières de ses emprunts, par des recettes commerciales. Celles-ci peuvent, par exemple résulter d'un péage acquitté par les usagers ou d'un péage fantôme (shadow toll ${ }^{7}$ ) acquitté par la puissance publique. Dans le cas d'un projet déficitaire, il est supposé que le déficit est compensé par la puissance publique : le niveau de subvention, déterminé sur la base d'une analyse coût - bénéfice établie ex ante, doit compléter les recettes attendues de sorte que soit assurée à l'opérateur la couverture des coûts complets ;

- Dans l'option dite “privée ", le mécanisme est identique, à ceci près que l'opérateur privé inclut dans ses charges la rémunération de ses capitaux propres et, donc, la réalisation d'un profit.

Les calculs de rentabilité financière sont, dans les deux cas, réalisés à prix constants.

Le taux de rentabilité interne propre au projet (TRI) est supposé identique selon que l'opérateur est public ou privé. Nous savons que cette hypothèse n'est pas nécessairement pertinente mais nous la retenons comme hypothèse de travail à ce moment de l'analyse.

\footnotetext{
${ }^{6}$ Cas particulier et ambigu car, officiellement, les dettes concernées (SEMCA et RFF) étant hors du champ des administrations publiques au sens de ce critère, l'Etat ne s'est pas privé de les laisser s'endetter, reportant à plus tard ses obligations de garant de la dette (soit par des subventions futures soit par une prolongation de la concession qui revient à différer les recettes publiques futures).

${ }^{7}$ Le shadow toll correspond à une gratuité du péage pour l'usager, mais la puissance publique compense cette gratuité en acquittant elle même ce péage. L'opérateur est ainsi incité a satisfaire au mieux la demande dès lors que ce péage fantôme est supérieur au coût marginal d'usage.
} 
Sur ces bases, l'opérateur public est supposé mettre en œuvre le projet si le TRI attendu peut couvrir le taux d'intérêt du marché augmenté d'une prime de risque prenant en compte les incertitudes associées à tout exercice d'évaluation financière de projet: incertitudes sur les coûts et sur les prévisions de trafic et de recettes. Pour fixer les idées, si les taux de long terme sont à $4 \%$ sur le marché financier et si la prime de risque est estimée à $4 \%$ également, l'opérateur public ne peut s'engager qu'avec un TRI au moins égal à $8 \%$. Pour toute valeur inférieure, une subvention compensatoire est requise afin que ce seuil soit atteint.

Pour le même projet, l'opérateur privé doit couvrir un taux d'intérêt du marché supposé identique plus la même prime de risque, mais il doit ajouter à cela une marge bénéficiaire. Disons, par exemple, $4 \%$ de plus. Cela signifie que pour toute valeur du TRI inférieure à $12 \%$, une subvention sera requise pour assurer l'équilibre financier du projet.

Observons que cette sollicitation du contribuable est théoriquement justifiée, que l'opérateur soit public ou privé, par des avantages externes au bilan financier du projet. Le fait que ces éléments soient "externes » par rapport aux comptes de l'opérateur, et même par rapport à la sphère marchande, n'empêche pas, bien entendu, qu'ils soient passibles d'un calcul économique.

En effet, si le bilan actualisé d'un projet est élargi à l'ensemble des coûts et avantages pour la collectivité, y compris divers effets externes, un taux de rentabilité interne peut être calculé. Il est généralement appelé taux de rentabilité socio-économique ou, plus simplement, économique (TRE). Ce n'est plus alors le seul point de vue du transporteur et de son compte de résultat qui est retenu, mais celui de la collectivité tout entière. Les pertes et avantages de tous les agents économiques sont ainsi évalués, telles, par exemple, les pertes de recettes nettes des modes concurrents ou les variations de surplus des usagers, ou encore les conséquences du projet sur la sécurité ou l'environnement ${ }^{8}$.

Les situations abondent, qui présentent des TRI insuffisants pour permettre un autofinancement du projet, mais des TRE qui justifient sa réalisation avec une part de financement public ou même un financement public intégral. Les transports publics urbains en site propre en sont un bon exemple. Il va de soi que ces situations sont d'autant plus fréquentes que les coûts sociaux sont mal internalisés.

Nous nous plaçons, dans l'analyse qui suit, dans des cas de figure où les TRE sont réputés justifier l'investissement mais où le TRI peut appeler une part de financement public. Les rentabilités attendues par l'opérateur, selon qu'il est public ou privé, correspondent alors à des zones du TRI initial du projet représentées sur la figure 1 ci dessous.

\footnotetext{
${ }^{8}$ Un groupe de travail présidé par Marcel Boiteux a proposé une première mise à jour des méthodes de calcul et des valeurs unitaires à prendre en compte pour les externalités en 1994, puis un deuxième rapport plus complet en 2001 (Cf. bibliographie).
} 
Figure 1 : Autofinancement et subventions

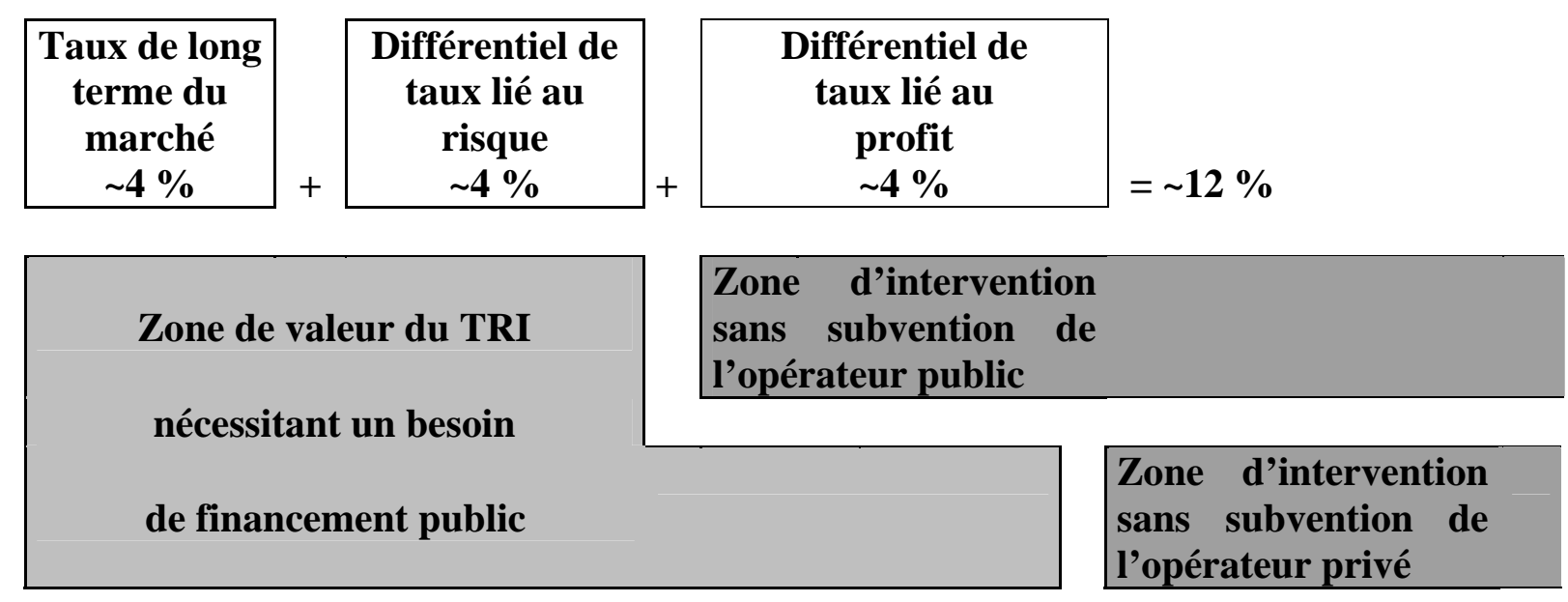

Plage de variation du Taux de Rentabilité Interne...........

Dans des circonstances concrètes, il va de soi que le défi pour l'opérateur privé peut être d'atteindre un TRI plus élevé grâce à une gestion plus efficiente du projet mais nous avons déjà indiqué que cet aspect des choses, quoique fondamental, n'est pas traité ici.

Sous les hypothèses retenues, cette présentation nous suggère que l'option "privée » peut être plus coûteuse pour les finances publiques que l'option «publique ». Elle reste cependant grossière car elle ne précise pas la relation entre le besoin de subvention et les TRI qui sont en cause. Il nous reste donc à établir cette relation.

\section{Une relation fondamentale}

Afin de formaliser cette relation, nous considérons un projet type impliquant un investissement de montant $C$, supposé réalisé sur une durée $d$, qui représente un nombre d'années au cours desquelles les dépenses sont supposées également réparties. A la mise en service, le bénéfice net dégagé par l'exploitation du projet est noté a et il est supposé croître annuellement d'un montant $b$.

Cela correspond à une chronique stylisée, mais somme toute classique ${ }^{9}$, des coûts et des bénéfices représentée sur la figure 2 . Si la mise en service est supposée réalisée à la date $\mathrm{t}=0$, la dépense annuelle entre les dates $-\mathrm{d}$ et 0 est de $\mathrm{c}=\mathrm{C} / \mathrm{d}$. A partir de la mise en service, le bénéfice dégagé est supposé de la forme $(\mathrm{a}+\mathrm{b} . \mathrm{t})$.

\footnotetext{
${ }^{9}$ Dans la pratique des recommandations officielles, les circulaires qui précisent les principes de calcul peuvent proposer des prévisions de demande considérées prudemment comme linéaires, ou encore des prévisions exponentielles jusqu'à une échéance donnée puis linéaires au delà. Le résultat net (bénéfice - coût) correspond alors au même type de fonction. Les calculs qui suivent se transposent sans difficulté avec une fonction exponentielle et les analyses qui en résultent n'en sont pas radicalement modifiées.
} 


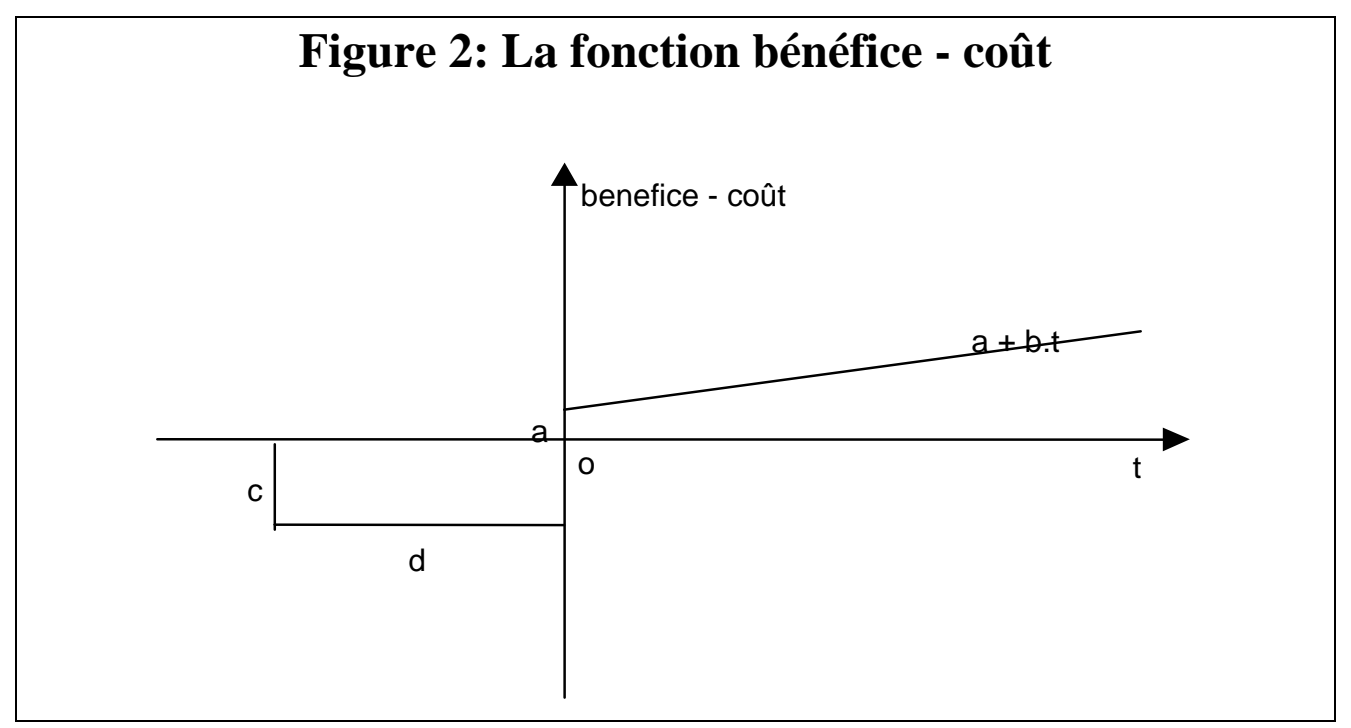

Le taux de rentabilité interne du projet (TRI), c'est à dire le taux d'actualisation qui annule sa valeur actualisée nette (VAN), est alors une fonction des quatre paramètres $\mathrm{C}, \mathrm{d}$, a et b. Il est à comparer au taux de rentabilité qu'un opérateur (public ou privé) est en droit d'escompter.

Nous utiliserons les notations suivantes :

$\alpha \quad$ est le taux d'actualisation utilisé pour calculer la valeur actualisée nette (VAN),

$\alpha_{0} \quad$ est le taux d'actualisation qui annule la VAN du projet, c'est à dire son TRI,

$\delta \quad$ est le supplément de TRI que la subvention apporte à l'opérateur,

$\tau \quad$ est le taux de subvention de l'investissement, soit la part de c financée par subvention.

Pour un taux d'actualisation $\alpha$, et un bilan actualisé calculé des dates $-\mathrm{d}$ à $\mathrm{T}$, la valeur actualisée nette du projet s'écrit :

$$
\mathrm{VAN}=\int_{-d}^{0}-\mathrm{c} \cdot \mathrm{e}^{-\alpha \mathrm{t}} \cdot \mathrm{dt}+\int_{0}^{T}(\mathrm{a}+\mathrm{b} \cdot \mathrm{t}) \cdot \mathrm{e}^{-\alpha \mathrm{t}} \cdot \mathrm{dt}
$$

Dans un souci de simplification des calculs, nous supposerons que l'actualisation est étendue à l'infini, ce qui est sans conséquence sur les résultats qui nous intéressent en raison du faible poids du futur lointain et, surtout de la convergence des fonctions intégrales de (1). Cette équation devient alors :

$$
\mathrm{VAN}=\left[\frac{\mathrm{c}}{\alpha} \mathrm{e}^{-\alpha \mathrm{t}}\right]_{-\mathrm{d}}^{0}+\left[-\frac{\mathrm{a}}{\alpha} \mathrm{e}^{-\alpha t}\right]_{0}^{+\infty}+\left[-\frac{-\mathrm{b} . \mathrm{t}}{\alpha} \mathrm{e}^{-\alpha \mathrm{t}}\right]_{0}^{+\infty}+\left[-\frac{\mathrm{b}}{\alpha{ }^{2}} \mathrm{e}^{-\alpha \mathrm{t}}\right]_{0}^{+\infty}
$$

ou encore :

$$
\mathrm{VAN}=\frac{1}{\alpha}\left[\mathrm{c}\left(1-\mathrm{e}^{\alpha d}\right)+\mathrm{a}+\frac{\mathrm{b}}{\alpha}\right]
$$

Le TRI du projet, $\alpha_{0}$, est alors donné par : 


$$
\mathrm{c}\left(1-\mathrm{e}^{\alpha 0 d}\right)+\mathrm{a}+\frac{\mathrm{b}}{\alpha_{0}}=0
$$

Un taux de subvention $\tau$ abaisse le coût annuel de construction $\mathrm{c}$ à $\mathrm{c}(1-\tau)$ et porte le TRI $\alpha_{0}$ à $\left(\alpha_{0}+\delta\right)$ de sorte l'équation (4) devient (4') :

$$
(1-\tau) \mathrm{c}\left(1-\mathrm{e}^{\left(\alpha_{0+\delta) d}\right.}\right)+\mathrm{a}+\frac{\mathrm{b}}{\alpha_{0}+\delta}=0
$$

Dont nous pouvons déduire l'expression du taux de subvention :

$$
\tau=1-\frac{\mathrm{a}\left(\alpha_{0}+\delta\right)+\mathrm{b}}{\mathrm{c}\left(\alpha_{0}+\delta\right)\left(\mathrm{e}^{(\alpha 0+\delta) d}-1\right)}
$$

L'élément important de cette fonction en regard des questions économiques qui nous intéressent est la relation entre $\tau$ et $\delta$. Cette relation dépend, évidemment, des paramètres c, $\mathrm{d}$, a, b et, bien sûr, $\alpha_{0}$, qui caractérisent l'économie du projet. Ces paramètres sont, en outre, liés entre eux par l'équation (4) qui définit le TRI du projet $\alpha_{0}$. Cela implique que si nous voulons représenter l'équation (5), il faudra figer certains de ces cinq paramètres et ne faire varier que ceux dont nous souhaitons exhiber le rôle. C'est, tout simplement, la technique classique des abaques.

Nous n'examinerons ici qu'un seul de ces abaques, suffisant pour illustrer notre propos et présenté sur la figure 3. Le coût annuel de construction c a été fixé à une valeur normée de 100, la durée de cette construction est fixée à 5 ans. L'accroissement annuel des avantages nets des coûts, $b$, est supposé égal à 1 . Cela revient à faire varier le TRI initial du projet $\alpha_{0}$ (ou encore a, puisque $\alpha_{0}$ ne dépend plus que de a, bénéfice net du projet à la date de mise en service). L'abaque ci-dessous présente ainsi la fonction 5 pour une série de valeurs de $\alpha_{0}$, entre $2 \%$ et $14 \%$ avec un pas de $0.4 \%$. Pour chacune de ces valeurs de $\alpha_{0}$, qui se lit en abscisse, chaque courbe exprime le taux de subvention nécessaire pour élever le TRI aux valeurs indiquées. 


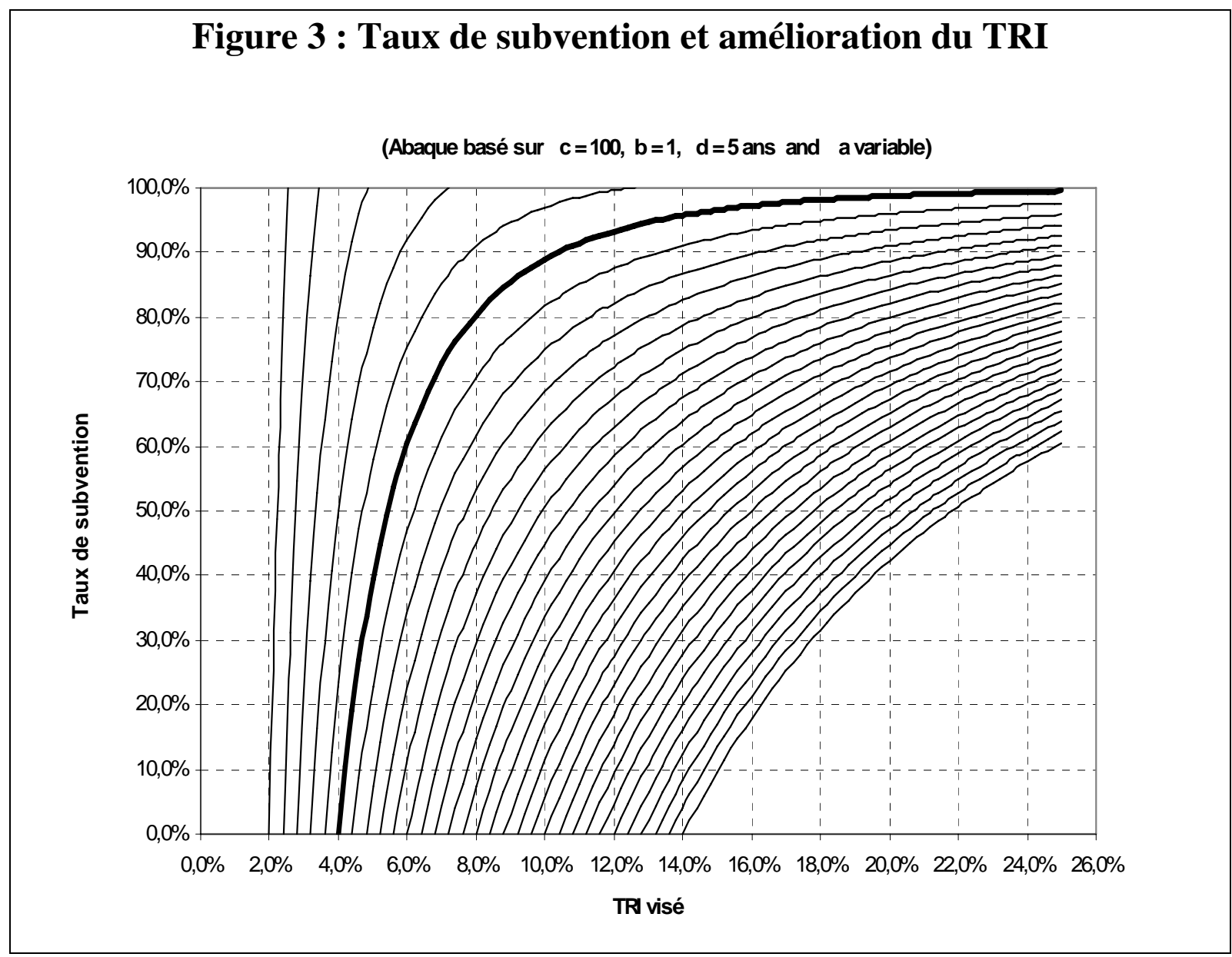

Bien entendu, ces fonctions ne doivent en aucun cas être considérées comme tout à fait générales en raison du jeu d'hypothèses qui nous a permis de les établir, qu'elles concernent les caractéristiques de la série chronologique des coûts et avantages du projet considéré ou les valeurs particulières retenues pour certains paramètres.

Toutefois, nous n'avons pas retenu des hypothèses extraordinaires. Nous pouvons d'autant mieux prendre en compte ce qui nous est suggéré par la forme de ces courbes que les abaques obtenus en faisant varier différemment (mais raisonnablement) les paramètres nous y autorisent. Au demeurant, les silhouettes de ces courbes sont liées à des propriétés de la dérivée seconde de la fonction représentée qui peuvent être aisément démontrées. Il en résulte des conséquences importantes quant au recours avec un partenariat privé.

\section{Concavité et paradoxe de la rentabilité financière}

Il est bien normal que le besoin de subvention soit une fonction croissante du taux de rendement interne qu'il s'agit d'assurer à l'opérateur. La pente de la courbe est, en outre, fortement décroissante. Cette concavité constitue un résultat contre-intuitif : cela signifie, en particulier, que les premiers écarts entre le TRI visé et le TRI de l'opération sont très coûteux : ainsi, un écart de quatre points entre ces deux valeurs, lorsque le TRI initial est de $8 \%$, implique un besoin de financement public du projet qui représente $45 \%$ de son coût.

Ce besoin est plus important encore pour des projets dont la rentabilité propre est plus faible. Si elle est de $4 \%$, par exemple, son amélioration de quatre points requiert un taux de subvention de $80 \%$ ! 
Cela signifie que l'effet de levier des finances publiques sur le rythme de réalisation des investissements est beaucoup plus sensible qu'on ne le soupçonne généralement au fait que l'on accorde ou non une priorité aux projets dont les TRI sont les plus élevés.

Lorsque ces taux sont proches du taux requis par l'opérateur public, le recours à un opérateur privé qui ne serait pas en mesure d'améliorer sensiblement la rentabilité de l'opération peut être coûteux, comme l'illustre l'exemple d'un passage de 8 à $12 \%$ évoqué cidessus.

La concavité des courbes a une autre conséquence importante : pour des projets de faible TRI initial, le passage d'un opérateur public à un opérateur privé a un faible coût marginal pour les finances publiques. Dans l'exemple également évoqué d'un TRI initial de $4 \%$, représenté par une courbe en gras sur notre abaque, aux $80 \%$ de taux de subvention nécessaires pour atteindre une rentabilité de $8 \%$, il suffit, pour le porter à $12 \%$ d'ajouter un taux de subvention de $13 \%$.

Ces résultats illustrent ce que nous avons appelé le paradoxe de la rentabilité financière ${ }^{10}$, qui peut s'exprimer ainsi : alors que le surcoût pour la puissance publique d'un recours à un opérateur privé par rapport à un opérateur public supposé aussi efficient est plus élevé lorsque la rentabilité d'un projet est proche de la rentabilité requise par l'opérateur public, ce surcoût est d'autant plus faible que le TRI initial est lui même faible.

Ce résultat rejoint l'observation, également paradoxale, selon laquelle le retour des entreprises privées dans le développement des grandes infrastructures se produit au moment où les projets qui restent à réaliser sont sensiblement moins rentables que ceux déjà en service. Le paradoxe théorique n'est évidemment pas l'explication du paradoxe empirique car nous avons vu que celui-ci a son histoire propre, mais il révèle que le mouvement de privatisation devrait susciter moins de difficultés de financement pour la puissance publique que ne le suggère une analyse trop sommaire.

Il reste évidemment à enrichir ces considérations des dimensions de la problématique du partenariat public - privé que nous avons éludées, à commencer par celle de l'efficacité respective des entreprises publiques et privées. Sans prétendre explorer en profondeur cette dimension pour des raisons qui seront précisées un peu plus loin, nous pouvons apercevoir sur l'abaque de la figure 3 quelques conséquences fortes de la concavité de la fonction de besoin de subvention sur la levée de l'hypothèse d'efficience égale des opérateurs publics et privés.

\section{La levée de l'hypothèse d'égale efficience}

On peut, en effet, imaginer que l'opérateur privé est capable d'assurer une meilleure rentabilité interne de l'opération, soit par une meilleure maîtrise des coûts d'exploitation (amélioration de a et de $\mathrm{b}$ dans la formule 4 qui détermine le TRI $\alpha_{0}$ ), soit par une économie sur les coûts d'investissement (abaissement de c), soit par de plus courts délais de construction (réduction de d) ou soit encore par une combinaison de ces facteurs de rentabilité. A titre de simple illustration, nous supposerons que le TRI initial $\alpha_{0}$ s'en trouve amélioré de $2 \%$.

Pour des valeurs variables de ce le TRI $\alpha_{0}$, nous obtenons alors des valeurs des taux de subvention reportées sur la figure 4 ci-dessous en conservant l'hypothèse évoquée dans la troisième partie selon laquelle le «TRI visé » est de $8 \%$ pour l'opérateur public et de $12 \%$ pour l'opérateur privé.

\footnotetext{
${ }^{10}$ Ce paradoxe a été précisé une première fois dans une publication antérieure (Bonnafous, 1999), mais ne reposait pas sur l'analyse mathématique présentée ici : des courbes proches de celles de la figure 3 avaient été établies sur la base de simulations empiriques produites par le modèle CALQUECO (Faivre d'Arcier, Mignot, 1998).
} 
Nous utilisons ainsi une configuration plus particulière encore que celle qui a permis d'établir l'abaque de la figure 3 : d'une part en fixant ces seuils pour les TRI visés, d'autre part en formalisant ainsi l'effet d'efficience. Pour autant, les courbes obtenues ne sont que la conséquence de la concavité de la fonction du taux de subvention.

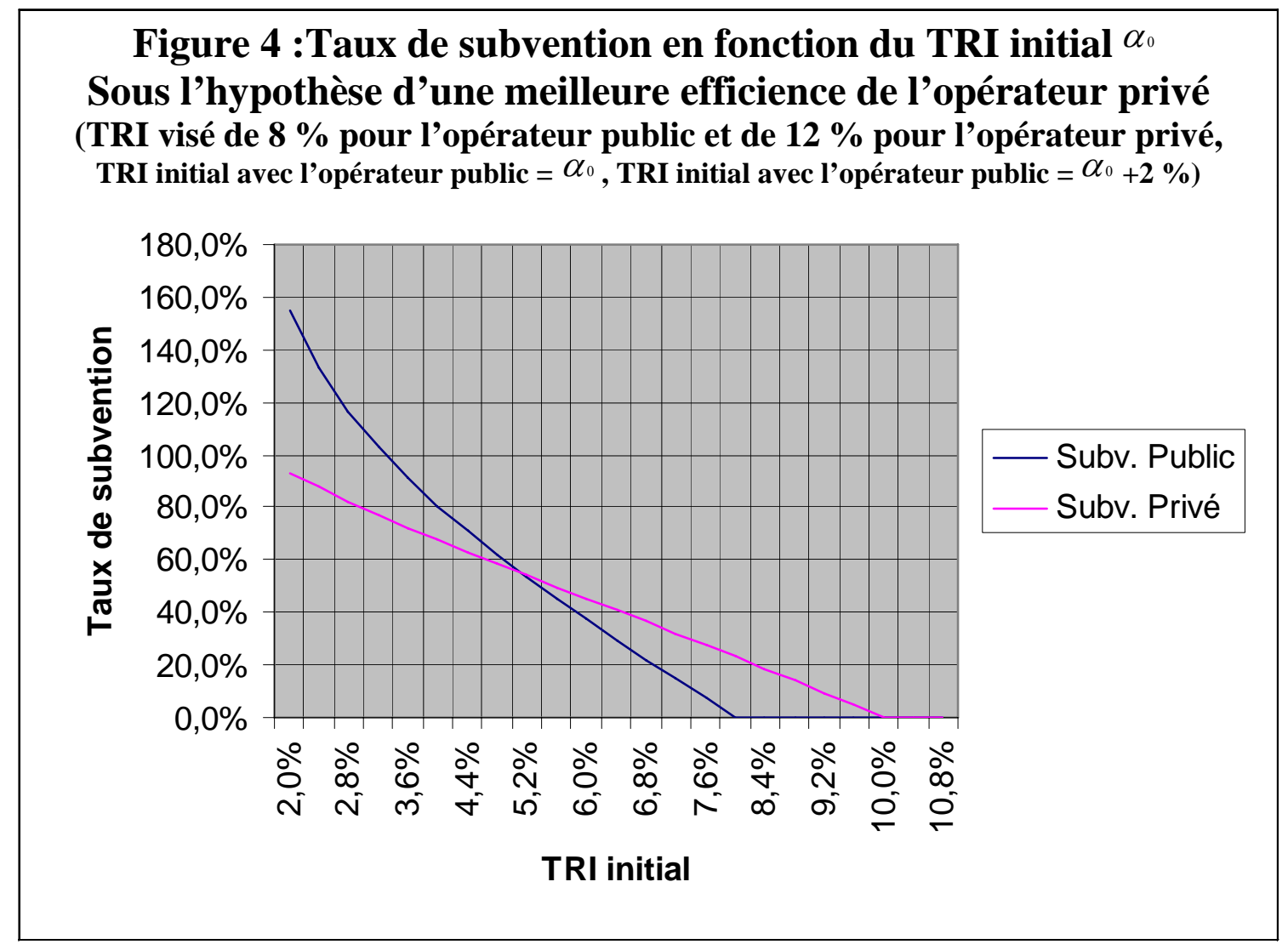

L'intérêt de ce graphique tient à ce qu'il nous suggère, dans la configuration particulière qu'il représente, que trois zones de valeurs du taux de rentabilité interne peuvent être distinguées. Elles correspondent à trois univers de choix du décideur politique relativement contrastés :

1) Dans la partie droite du graphique, pour des rentabilités du même ordre ou supérieures à celle que vise l'opérateur public, les finances publiques ne peuvent que perdre lors du recours à l'opérateur privé. Lorsque la perte est limitée, ce recours peut être cependant justifié par le surplus global de productivité dont bénéficie l'économie dans son ensemble du fait du différentiel d'efficience ;

2) Dans la partie gauche du graphique, pour des rentabilités très faibles, le différentiel d'efficience joue un rôle considérable, mais nous approchons alors du cas de figure dans lequel le rendement social du projet peut être insuffisant et conduire à le remettre en cause ou, du moins, à remettre en cause sa consistance ${ }^{11}$. Cependant, si sa rentabilité socio-économique justifie sa réalisation, la concession à un opérateur privé sera moins coûteuse pour les finances publiques ;

3) Entre ces deux zones, il y a quelque part un «basculement » pour une valeur particulière ${ }^{12}$

\footnotetext{
${ }^{11}$ Dans le cas des autoroutes, par exemple, il peut être alors plus judicieux de renoncer à une autoroute à péage au profit d'une liaison à deux fois deux voies aux caractéristiques moins exigeantes et moins coûteuses ne seraitce qu'en raison de la possibilité d'utiliser tout ou partie de la route traditionnelle.

${ }^{12}$ Pour l'anecdote numérique cette valeur s'établit à 5,2\% pour $\alpha_{0}$ dans le cas de simulation retenu.
} 
de $\alpha_{0}$ en deçà de laquelle le recours à un opérateur privé réduit la dépense publique. En ce cas, le critère du rendement social et celui de l'économie de fonds public convergent pour désigner l'opérateur privé comme le meilleur choix collectif.

Il convient de souligner que l'existence de ce basculement n'est pas une conséquence nécessaire de la concavité de la fonction de besoin de subvention : il existe évidemment des valeurs des paramètres pour lesquelles cette fonction est en tout point supérieure dans le cas de l'opérateur privé. Le paradoxe de la rentabilité financière n'implique donc l'existence d'un point de basculement que pour un sous-ensemble des valeurs possibles des paramètres a,b,c et $\mathrm{d}$, mais aussi les TRI ciblés par les deux types d'opérateur et, bien entendu, du différentiel d'efficience dont le niveau est évidemment décisif.

\section{Conclusion}

Si les opérateurs privés ne sont pas plus efficients que les opérateurs publics, il est toujours plus coûteux pour l'Etat de concéder une infrastructure publique à une entreprise privée. Cependant, ce surcoût pour les finances publiques est d'autant plus faible que la rentabilité financière du projet est elle-même faible.

Si les opérateurs privés sont plus efficients, ce surcoût peut devenir un gain, d'autant plus sûrement que la rentabilité financière du projet est faible. Il n'est donc pas anormal que des opérateurs privés se voient confier des projets très peu rentables, cette option pouvant à la fois abaisser la dépense publique et élever le rendement social du projet.

Cependant, cette transformation d'un surcoût en un gain, ce basculement, n'est pas automatique mais requiert des combinaisons de valeurs des paramètres qui ne sont pas quelconques. Les conditions de l'existence d'un point de basculement restent à explorer probablement d'une manière différente de celle que nous avons utilisée précédemment. En introduisant un effet d'efficience supposé améliorer de $2 \%$ le TRI initial de l'opération quelle que soit sa valeur, nous procédons à une homothétie bien téméraire : que cet effet résulte, par exemple, d'une plus grande rapidité des travaux ou d'une meilleure efficacité commerciale, il y a peu de chance pour qu'il élève d'un même niveau la valeur du TRI. Nous n'avons procédé à cet artifice qu'en raison du nombre des paramètres en jeu qui rend peu efficace l'usage des abaques.

Pour redonner à l'usage des simulations et de leur représentation en abaque leur efficacité, il conviendrait de réduire les gammes des paramètres, c'est à dire de déterminer leurs plages de variation qu'il est pertinent de combiner. Les recherches empiriques nécessaires à cette détermination restent à faire, qui permettraient de décliner les jeux de simulation utiles. Gageons que ces déclinaisons seraient bien différentes selon les conditions économiques, financières et sociétales propres à chaque politique nationale ou régionale et à chaque secteur d'activité, que ce soit dans le champ des transports ou au delà.

\section{REFERENCES BIBLIOGRAPHIQUES}

Baird, A.J. « Trend in Port Privatisation in the World Top 100 Containers Ports. » $9^{\text {th }}$ WCTR, Seoul, July.2001, 21p.

Bonnafous, A. et Crozet, Y. «Evaluation, dévaluation ou réévaluation des lignes à grande vitesse ? » Les Cahiers Scientifiques du Transport, n³2, 1997.

Bonnafous, A. «Infrastructures publiques et financement privé : le paradoxe de la rentabilité financière. ", Revue d'Economie Financière, n51, 1999.

Bonnafous, A. "Transport Infrastructures and Private Financing: a Profitability Rate Paradox. » $9^{\text {th }}$ WCTR, Seoul, July.2001, 9p. 
Budin, K.J. and Thompson, L.S. «Directions of Railway Reform », Rail International Schienen der Welt, sept.-oct. 2001, pp. 30-45.

Cohen, Y. (1991) «California's Private Infrastructure Initiative. » Journal of Transport Economics and Policy n²5, 1991

Commissariat Général du Plan. Transports : pour un meilleur choix des investissements. Rapport du groupe présidé par Marcel Boiteux, Documentation Française, 1996.

Commissariat Général du Plan. Transports : le prix d'une stratégie, Rapport de l'atelier présidé par Alain Bonnafous, 2 vol., Documentation Française, 1997.

Dewenter, K.L. and Malatesta, P.H. "State-Owned and Privately Owned Firms : An Empirical Analysis of Profitability, Leverage, and Labor Intensity. » American Economic Revew, March 2001, 91(1), pp.320-334.

Faivre d'Arcier, B. and Mignot, D. « Using Economic Calculation as a Simulation Tool to Assess Transport Investments $», 8^{\text {th }}$ WCTR, Anvers, July 1998, 14p.

Gomez-Ibanez, J.A. and Meyer, J.R. Going Private, Brooking Institution, Washington D.C., 1993.

Mills, F. «Public Infrastructure : Private Ownership or Contracting Out. » Working Paper $\mathrm{n}^{\circ} 18$, Center for Microeconomic Policy Analysis, University of Sydney, 1996. 